\title{
Testing Flight Software on the Ground: Introducing the Hardware-in-the-Loop Simulation Method to the Alpha Magnetic Spectrometer on the International Space Station
}

\author{
Wenhao Sun ${ }^{\mathrm{a}, *}$, Xudong Cai ${ }^{\mathrm{b}}$, Qiao Meng ${ }^{\mathrm{a}}$ \\ ${ }^{a}$ Southeast University, Nanjing 210096, China \\ ${ }^{b}$ Massachusetts Institute of Technology, MA 02139-4307, U.S.A.
}

\begin{abstract}
Complex automatic protection functions are being added to the onboard software of the Alpha Magnetic Spectrometer. A hardware-in-the-loop simulation method has been introduced to overcome the difficulties of ground testing that are brought by hardware and environmental limitations. We invented a time-saving approach by reusing the flight data as the data source of the simulation system instead of mathematical models. This is easy to implement and it works efficiently. This paper presents the system framework, implementation details and some application examples.
\end{abstract}

Keywords: Alpha Magnetic Spectrometer(AMS), Hardware-in-the-Loop Simulation, Embedded Software Test

\section{Introduction}

The Alpha Magnetic Spectrometer (AMS) is a particle physics experiment mounted on the International Space Station (ISS) [1, 2]. It was designed to measure antimatter in cosmic rays and search for evidence of dark matter.

During the continuous operation of AMS, scientists of the AMS collaboration monitor the instrument status 24/7. 5 Real-time monitoring allows issue resolution, such as communication anomalies or hardware failures, as soon as possible.

However, ground monitoring is not $100 \%$ reliable due to communication outages. To prevent potential sudden damage, more and more automatic protection mechanisms are being added into the onboard software as operational experience grows.

Automatic protection functions define some conditions, and once the conditions are triggered, the control system would take corresponding actions.

Consider the following scenario: a function is designed to monitor a group of temperature sensors, and it is supposed to do something if temperature alarms are detected on certain sensors. If the trigger logic is simple, e.g. only 1 sensor is taken into account, the functions would be easy to implement; otherwise, e.g. a configurable set of 5 sensors are involved, the implementation would not be that obvious and the validity needs to be tested rigorously.

To perform such a test, we need to generate the triggering conditions that might be some sensors getting abnormal temperatures, but we have to face the fact that not all devices are installed in the ground test system. Furthermore, it is not easy to raise or lower the temperature whenever we want.

In order to overcome the difficulties brought by hardware and environmental limitations, we introduced the 20 hardware-in-the-loop (HIL) method. Hardware-in-the-loop simulation is a technique that is widely used in the development and test of real-time control systems [3]. It connects the real controller in the simulation loop and virtual devices which interact with the controller.

In this research, we applied a time-saving approach and built the HIL system for AMS flight software test: based on the knowledge of AMS operations (Section 2), we built a general behavior model for the virtual devices and invented a language to describe different devices (Section 3); instead of building mathematical models for the simulated

${ }^{*}$ Corresponding author. Tel.: +86-159-5051-3326

Email address: wenhao_sun@126.com (Wenhao Sun) 
environment, we set up a data flow to feed the simulation system with the real-time flight data of AMS and made it possible to reproduce space environment on the ground (Section 34); customized data derived from the flight data generates various conditions as expected (Section 344); we compared our approach with typical HIL schemes, and summarized how to take advantage of our idea and build HIL systems to assist maintenance of complex systems (Section 5).

\section{AMS operations}

AMS consists of a series of detectors that are used to determine various characteristics of the radiation and particles as they pass through. Figure 1 shows the main components: Transition Radiation Detector (TRD), Time of Flight counter (ToF), silicon tracker, permanent magnet, Anti-Coincidence Counter (ACC), Ring Imaging CHerenkov detector (RICH), and Electromagnetic CALorimeter (ECAL).

Signals from these detectors are processed by the electronics. Data is transmitted under a set of communication protocols. All processes are managed by real-time software onboard the controllers.

Manual control from the ground is possible only when we have good communications with the ISS. Hence communication outages pose potential risks.

A solution is to add automatic procedures in the onboard software, where the triggering conditions are undoubtedly essential and should be verified and validated.

\subsection{AMS electronics}

The AMS electronics has 650 boards and about 300,000 data channels. This large amount of electronics could be divided into 2 major parts, the Data Acquisition (DAQ) System and the Slow Control System, besides which the

Main Data Computer (MDC) deals with all commands and data on the top layer. Figure 2 shows the basic topology of the system [4, 5, 6].

Node is an abstract concept here. In general, a node refers to a processor unit and the devices under its control. The processor could be a digital signal processor (DSP) in the DAQ System or a microcontroller unit (MCU) in the Slow Control System.

\subsection{Communication protocols and data formats}

Communications inside AMS follows the master-slave principle: high-layer nodes are masters and low-layer nodes are slaves accordingly. MDC is the master of the entire AMS electronics, in other words, all the other nodes are slaves of it.

Two main communication protocols are applied: AMSWire protocol (customized on the basis of the IEEE 1355 SpaceWire Standard) in the DAQ system and the Controller Area Network (CAN) protocol in the Slow Control System [7]. For each protocol, an encapsulation format, namely AMSWire packet and CAN packet, was designed to carry AMSBlocks, the standard format for AMS data processing.

Since AMS is a payload of the ISS, protocols and data formats used by the ISS are involved in the AMS data flow as well.

\subsection{Flight operations}

The MDC software automatically manages almost every aspect of regular operations: data acquisition, storage management, communications, monitoring, etc. If some new features are required for automatic control, the MDC software must be updated.

As slaves of the MDC, nodes in DAQ and Slow Control systems work in "slave" mode: get instructions, do something, and send replies. These nodes send control signals and collect data if and only if they get explicit instructions from their masters. 


\subsection{Ground operations and potential risks}

MDC is unable to decide the execution time for every possible operation, while ground commands tell it what to do occasionally. However, not every command arrives in time.

On one hand, ground commands would be sent out only when the communication link works well; on the other hand, delay of telemetry data postpones the reactions more or less.

The inevitable delay of ground commands brings potential risks, especially when AMS is facing environmental changes in space. Consequently, we try to migrate more activities to MDC and reduce risks.

\subsection{Software updates and ground tests}

The AMS collaboration has accumulated a wealth of operational experience since the installation of AMS took place in May 2011. From time to time, people expect more features of the MDC software which would reduce the complexity of ground operations.

When a new version is ready to go, the software needs to be tested before we upload it to AMS. Real-time control software should be tested on certain hardware, and the Flight Simulator, a spare of the AMS electronics, provides so the facilities.

The Flight Simulator is available in the lab. It is equipped with some nodes and sensors, but not all. On the flight simulator, we can update MDC software and test newly added functions.

The hardware and environmental limitations, however, make some tests difficult: if a function relies on either return values from a component that is missing on the Flight Simulator, or changes of the external environment that the laboratory environment will not provide, the tests cannot proceed.

\section{HIL simulation for AMS software test}

The HIL system in this research was designed to test AMS software, specifically the MDC software. Functions that are triggered by slave node anomalies cannot be tested during normal operation, and the HIL system would provide these conditions.

\section{3.1. Simulate a node}

As there are hundreds of nodes, the central question of the task is how to simulate each different node in a relatively easy way.

First of all, we would like to make it more clear how a real node works. Figure 3 illustrates the behavior patterns of a slave node: a slave is controlled by the commands, or "requests", from the master; and the execution results, or "replies", are read back. As for a specific execution process, the behavior differs due to the variety of node functions, which makes it hard to simulate inner mechanisms of every node. In addition, devices are not isolated and they may influence each other. For example, a cooling loop parameter may determine the values read out by many pressure and temperature sensors.

It is not feasible to simulate all nodes with mathematical models, but if we ignore the details inside a node, from the standpoint of the master, the behavior of a node could be considered as a black box with a "request" input and a "reply" output. As long as we can analyze requests and provide replies, a general model would be available. It is not easy to generate the reply data, but fortunately, we have the flight data, the real-time data from AMS in space, which contains comprehensive information of instruments including the status changes.

We adopted the following solution: build a general behavior model describing the request-reply feature; prepare the reply data on the basis of the existing flight data; customize the model for each node and define how to understand requests and generate replies; connect the simulated nodes to a real MDC, referred as a hardware-in-the-loop simulation, and the MDC could get the test environment needed.

\subsection{Hardware-in-the-loop system}

Figure 4 shows the structure of the HIL system in this research: a personal computer (PC) with an interface device is connected to the electronics. Simulation software running on the PC constructs virtual nodes and communicates with the real world via the interface device which deals with both AMSWire and CAN protocols. 


\subsubsection{Virtual world}

The virtual world of the HIL system refers to the simulation software. The software builds some virtual nodes as required, defines the behavior of each node, and specifies reply data as the execution results. Data transmission is based on a communication port of the PC.

Figure 5 is a diagram of the simulation software:

1. Before a test, we construct a simulation database by extracting or modifying the flight data of AMS;

2. When the simulation software is running:

(a) A hardware driver manipulates the interface device and processes AMSWire/CAN packets;

(b) The packets are converted to/from AMSBlocks, and the information contained in AMSBlocks is used by a general behavior model of AMS nodes;

(c) The node model analyzes each AMSBlock, and determines what to reply;

(d) If needed, reply data could be read from the simulation database.

\subsubsection{Interface}

As a bridge between the real and virtual worlds, the interface device plays an important role.

Currently the interface is a device called "EPP-Box", which was developed to test the AMSWire and CAN protocols of the AMS electronics. Because of its ability to handle both protocols, the EPP-Box is now used as the transit point of data.

The structure of EPP-Box is presented in Figure 6 it communicates with a computer via the Enhanced Parallel Port (EPP), and it is equipped with 4 AMSWire ports and 2 CAN ports.

Since AMSWire is a point-to-point protocol and CAN is a bus protocol, an EPP-Box is capable of acting as multiple nodes: an AMSWire port serves as an AMSWire node and all its slaves; a CAN port listens to a CAN bus and behaves as any slave node(s) on that bus.

\subsubsection{Real world}

As mentioned, the flight simulator provides facilities for the MDC software test. Naturally, the real world of the HIL system is a part of the flight simulator.

In the HIL system, some of the real hardware is involved: a real MDC is essential for a HIL test; for the nodes that do not affect the test if they give out data of the laboratory environment, the real hardware can be connected in the simulation system, as the "Real" nodes in Figure 4

\subsection{General behavior model of virtual nodes}

The simulation software should be functional and flexible. Rather than require the users to define the node behaviors with $\mathrm{C} / \mathrm{C}++$ codes, we designed a descriptive language to simplify the customization.

\subsubsection{Behavior model}

We abstracted the behavior model (see Figure 7) based on the knowledge of AMS electronics: Node has a 9-bit NodeAddress and supports DataTypes; DataType is a 5-bit operation code and stands for at least 1 Command, a specific operation; Command has a read/write attribute and may carry some information about Requests and Replies; Request describes the filter criteria for the request; Reply specifies how to generate the reply data.

\subsubsection{Descriptive language}

The object-oriented paradigm has been applied in the descriptive language design.

We defined a set of keywords and the corresponding syntax, describing entities and attributes: "NODE" names a node and starts the entity; "ADDR" sets the node address; "DT" defines a data type as a member of NODE and sets the operation code; "RD/WR" means a command with read/write attribute, and it is a member of DT; "RQ..." are optional rules defining how a command checks request arguments and decides to make responses or not; "RP . .." are required rules defining how a command gets reply data.

The sample code in Figure 8 illustrates the usage of the descriptive language.

To let programs understand the descriptive language, we made a parser with Flex \& Bison [8]. To get the simulated nodes working properly, we constructed 3 important classes, namely Node, DataType and Command, to implement the interfaces provided in the descriptive language. 


\subsection{Simulation data flow}

Once we have virtual nodes of the electronics, how to make them provide data of various physical quantities?

The data flow in Figure 9 presents how the data is processed: flight data from AMS on the ISS is recorded in the flight database; the simulation database is constructed by extracting and modifying the flight data; the simulation software would be able to get all kinds of data from the simulation database; and finally the output of the MDC is recorded in the output database.

\subsubsection{Flight data}

The flight data is transmitted via the data link of ISS and stored in the form of AMSBlocks.

Flight data provides the operation records of MDC. From this database, we can find almost all parameters of AMS at a specific time, so it is often used for study of the running status of AMS.

Now that we need all information of a fully operational AMS, the flight database is the best data source.

\subsubsection{Simulation data}

To prepare for a simulation test, we can extract some data from the flight database, modify slightly if needed, and finally build the simulation database.

By Extraction. Depending on the required system configuration, we need to select a period of flight data and copy it to the simulation database. We made a tool to accomplish the task. It sifts through a period of flight data, decodes and classifies the AMSBlocks, and splits them into separate data files.

By Modification. Flight data makes it possible to reproduce situations in space, but for the test purpose, we still need some "abnormal" signals as the triggering conditions. In most cases, a single software function cares about a certain subsystem only. So we leave most of the flight data untouched, make modifications to a small part of the data files, generating extremely high/low values or errors like read failures. Each device may have specific data definitions, which makes it hard to analyze all the information carried in AMSBlocks. Rather than develop complex tools to modify the data files, we edit the binary data directly at the moment.

\subsubsection{Output data}

As soon as the anomalies are detected by the MDC, execution status of the function under test ought to be observed somehow.

Taking advantage of the Flight Simulator, we are able to obtain the output of MDC directly. Moreover, the HIL system records the received commands which reflect the MDC activities as well.

\subsection{Run a simulation}

Before a HIL test, the system should be configured properly. The procedure to run a simulation is as follows:

1. Understand the software functions under test, find out all the cases that need to be tested and the expected output for each case;

2. Build the simulation database and prepare the description files defining the simulated nodes;

3. Run the simulation software;

4. Make sure the hardware is connected correctly;

5. Start up the system;

6. For each case, observe the MDC operations, compare with the expected output, and make judgments whether the functions work well or not.

7. Complete the test if every case turns out OK, otherwise give feedback to the software developer with the test details.

Figure 10 demonstrates output of the simulation software: two nodes were simulated and a "ping" command was processed. 


\section{Case study}

The HIL system has been used to test the most resent versions of AMS onboard software. Three cases are presented below to illustrate how the system benefits the software test.

The background for all the examples is as follows: the Tracker Thermal Control System (TTCS) maintains the temperature stability of Tracker by pumping carbon dioxide $\left(\mathrm{CO}_{2}\right)$ two-phase loops [9] (see Figure 11]; TTCS monitors a series of temperature sensors and controls pumps by which the cooling liquid is pumped out; new software functions are being developed to help investigate the performance of TTCS; each temperature sensor has warning and alarm limits for high and low temperatures; pump speed has an upper limit and a lower limit; abnormal temperature or pump speed ought to be detected; MDC will take some actions if the anomalies are detected.

\subsection{Reproduce space environment}

The first step to perform a simulation test is to configure the flight simulator as the HIL system. Sensor signals are supposed to reflect the space environment after the simulation starts.

On the flight simulator, the Tracker Thermal Control Electronics (TTCE), the controller of TTCS, is mounted properly. As for the sensors, some of the temperature sensors are available, while the pump speed sensors give zero values because no real pumps are installed in the lab.

Relying on the flight data, we can reproduce the space data on the simulated TTCE. In Figure 12, we present the data from three sensors to explain the process.

In the first hour, the TTCE gave information of the laboratory environment. Available temperature sensors (like the first sensor) read out room temperature data, whose fluctuation was brought by the air conditioner; unavailable temperature sensors (like the second sensor) had no data, or to be exact, they gave out "invalid" data; pump speed sensors (like the third sensor) had zero values only.

In the following three hours, the simulated TTCS took the place of the real TTCE. For the first sensor, room temperature was replaced by the temperature in space, which changed gradually as the ISS flies around the Earth (the period is about 1.5 hours per orbit); for the second sensor, the "invalid" data turned "valid"; and for the pump speed sensor, the speed became 5000 RPM.

This example indicates that we are able to make the flight simulator "experience" the space environment. In case the software under test is designed for something that has ever happened in space, we may simply load the data of that period and see the reactions of the software.

\subsection{Generate different conditions}

The triggering conditions are most likely something that has never happened before. In this case, we can generate required conditions manually.

With the "functional" TTCS we got in the previous example, we started to produce a single triggering condition: an abnormal temperature or pump speed.

Anomalies could be classified into various types. A single temperature sensor may have 6 states: normal temperature; high temperature warning; high temperature alarm; low temperature warning; low temperature alarm; read failure. Accordingly, a pump speed sensor has 3 states: normal speed; high speed (over the upper limit); low speed (under the lower limit).

To meet possible needs of the triggering conditions, we generated different kinds of data. In principle, warnings and alarms are similar. So we generated high temperature, low temperature and invalid data for a temperature sensor; and applied high speed and low speed to a pump speed sensor.

Figure 13 shows the results: in Figure 13a, Temperature Sensor 1 was changed to 4 states, and in the meanwhile Temperature Sensor 2 gave flight data as reference; in Figure 13b. Pump Speed Sensor 1 had 3 states, and Pump Speed Sensor 2 also gave flight data, but as we usually keep only one pump running in space, the data was a constant zero.

In this example, we changed the status of single sensors without touching the others, which means we are ready for more complex triggering conditions. 


\subsection{Generate combined conditions}

Sometimes the automatic protection functions are called only when a series of conditions are satisfied.

In addition to the previous case, we could combine anomalies to get more complex triggering conditions. The following example demonstrates such a scenario: there are tens of temperature sensors in TTCS, and a function is designed to monitor two of them; only if both of the two sensors get anomalies, the function will throw some alarm signals; anomalies from other sensors are ignored.

To test such a function, we combined states of the specified two sensors and also involved other sensors. Figure 14 presents a part of this test: the first two sensors were required by the function, while the third was not; we combined the anomalies of the first two sensors, only when both of them got high temperatures or read failures, the function gave alarms; anomalies from the third sensor did not induce alarms.

This example was one of the tests we have performed for the recent versions of MDC software. With the HIL system, we easily simulated hundreds of combined conditions. More importantly, even if we simulate devices reading voltage, current, pressure or any other variable, exactly the same method will work well with no additional effort for any particular physical quantity.

\section{Discussion}

The hardware-in-the-loop simulation technique allows testing a controller with simulated environment instead of a real plant, which improves the development of the systems with restrictions on cost, duration, safety, and feasibility. So far, HIL simulation has been used in various disciplines, such as automotive systems [10, 11], power electronics [12, 13], robotics [14, 15], unmanned vehicles [16, 17], and so on.

When analog signals are involved, the simulated devices are usually built with mathematical models which calculate the physical quantities in real-time [18, 19].

Building models costs time and effort, and the difficulty grows dramatically as the complexity grows. Even though people come up with new ideas to simplify the model construction [20, 21], building an accurate model for all the devices under the control of MDC is still not feasible. Had we spent time and effort on mathematical models, we would hardly have simulated the space environment in every aspect.

Given that not many variables would be simultaneously used to generate triggering conditions, a reasonable idea is to utilize the flight data and make slight modifications. As a consequence, we invented the method applied in this research, which skips the mathematical model construction and uses a database instead.

A typical scheme of HIL simulation is shown in Figure 15 in the real world, real hardware of the controller is connected to receive data from simulated sensors and send commands to simulated actuators; and in the virtual world, models of the actuators and the sensors determine the behaviors of simulated devices, while a mathematical model simulates the physical process affected by the environment and also the operations of actuators.

For comparison, Figure 16 shows the HIL scheme of this research: the hardware and behavior models remain, but the mathematical model is replaced by "Simulation Database" and "Check Operations", which could be implemented much more easily. This design satisfies our current needs with less effort.

We believe this approach would be especially effective for maintenance of complex systems like AMS. Such systems have various devices to be simulated and it is not feasible to build a full set of mathematical models. The design in this research avoids mathematical models and splits the device model into a general behavior model and a database containing various data. With recorded data, a similar HIL system could be easily built. It is flexible to change the status of virtual devices by editing data, and more importantly, it is not a hard job so people can focus on the simulation itself rather than spend too much time on simulation tools.

However, the current solution has room for improvement. Notice that the simulation loop is closed in Figure 15 but open in Figure 16 The structural difference implies the open-loop scheme has less feedback features. In some cases, we may need closed-loop features to simulate dynamic processes. For instance, when the actuators get some commands, the simulated world is supposed to switch to another configuration. To satisfy this kind of demands, we propose to replace the "Check Operations" part by a "Dynamic Configuration" module (see Figure 17), which reconfigures the simulated devices, particularly the database, according to the commands from actuators. 


\section{Conclusion}

In this paper, we presented a time-saving method to implement hardware-in-the-loop (HIL) simulation for complex in traditional methods.

This method has been proved effective and efficient in the tests of AMS flight software. For further demands, we may improve the feedback features in the near future.

\section{Acknowledgments}

This research was supported by Southeast University (China), the China Scholarship Council, and the U.S. Department of Energy.

\section{References}

[1] A. Kounine, The alpha magnetic spectrometer on the international space station, International Journal of Modern Physics E 21 (08) (2012) 1230005 .

[2] S. Ting, The alpha magnetic spectrometer on the international space station, Nuclear Physics B-Proceedings Supplements 243 (2013) 12-24.

[3] M. Bacic, On hardware-in-the-loop simulation, in: Decision and Control, 2005 and 2005 European Control Conference. CDC-ECC'05. 44th IEEE Conference on, IEEE, 2005, pp. 3194-3198.

[4] V. Commichau, H. Broeker, C. Camps, G. Fluegge, K. Hangarter, M. Capell, A. Koulemzine, A. Lebedev, S. Xu, K. Kim, J. Yang, G. Kim, et al., The slow control system for the alpha magnetic spectrometer (ams-02) on the international space station, Proceedings of the ICALEPCS2003, Gyeongju, Korea (2003) 96-98.

[5] A. Lebedev, The ams-02 electronics system, in: International Cosmic Ray Conference, Vol. 3, 2005 , p. 377.

[6] F. Spada, The ams-02 electronics system, in: Astroparticle, Particle and Space Physics, Detectors and Medical Physics Applications, Vol. 1, 2006, pp. 989-993.

[7] X. Cai, On-board computing system for ams-02 mission, in: International Cosmic Ray Conference, Vol. 3, 2005 , p. 373.

315 [8] J. Levine, Flex \& Bison: Text Processing Tools, O’Reilly Media, Inc., 2009.

[9] G. Alberti, A. Alvino, G. Ambrosi, M. Bardet, R. Battiston, S. Borsini, J. Cao, Y. Chen, J. van Es, C. Gargiulo, et al., Active co2 two-phase loops for the ams-02 tracker, Aerospace and Electronic Systems Magazine, IEEE 29 (4) (2014) 4-13.

[10] I. Kendall, R. Jones, An investigation into the use of hardware-in-the-loop simulation testing for automotive electronic control systems, Control Engineering Practice 7 (11) (1999) 1343-1356.

[11] H. K. Fathy, Z. S. Filipi, J. Hagena, J. L. Stein, Review of hardware-in-the-loop simulation and its prospects in the automotive area, in: Society of Photo-Optical Instrumentation Engineers (SPIE) Conference Series, Vol. 6228, 2006.

[12] B. Lu, X. Wu, H. Figueroa, A. Monti, A low-cost real-time hardware-in-the-loop testing approach of power electronics controls, Industrial Electronics, IEEE Transactions on 54 (2) (2007) 919-931.

[13] M. O. O. Faruque, V. Dinavahi, Hardware-in-the-loop simulation of power electronic systems using adaptive discretization, Industrial Electronics, IEEE Transactions on 57 (4) (2010) 1146-1158.

[14] J. De Carufel, E. Martin, J.-C. Piedbœuf, Control strategies for hardware-in-the-loop simulation of flexible space robots, in: Control Theory and Applications, IEE Proceedings-, Vol. 147, IET, 2000, pp. 569-579.

[15] A. Martin, M. R. Emami, An architecture for robotic hardware-in-the-loop simulation, in: Mechatronics and Automation, Proceedings of the 2006 IEEE International Conference on, IEEE, 2006, pp. 2162-2167.

[16] D. Jung, J. Ratti, P. Tsiotras, Real-time implementation and validation of a new hierarchical path planning scheme of uavs via hardware-inthe-loop simulation, in: Unmanned Aircraft Systems, Springer, 2009, pp. 163-181.

[17] A. Hwang, S. Yoon, T.-Y. Kim, D.-Y. Kwon, C. Choi, H. Cho, Verification of unmanned underwater vehicle with velocity over 10 knots guidance control system based on hardware in the loop simulation, in: OCEANS 2009, MTS/IEEE Biloxi-Marine Technology for Our Future: Global and Local Challenges, IEEE, 2009, pp. 1-5.

[18] M. Karpenko, N. Sepehri, Hardware-in-the-loop simulator for research on fault tolerant control of electrohydraulic actuators in a flight control application, Mechatronics 19 (7) (2009) 1067-1077.

[19] Y. Reddy, A. Ramsesh, K. Raju, Y. Kumar, A novel approach for modeling and simulation of hybrid power systems using plcs and scada for hardware in the loop test, in: Sustainable Energy and Intelligent Systems (SEISCON 2011), International Conference on, IET, 2011 , pp. $545-553$

[20] J. A. Ferreira, J. E. De Oliveira, V. A. Costa, Modeling of hydraulic systems for hardware-in-the-loop simulation: A methodology proposal, in: Proc. Int. Mechanical Eng. Congress \& Exposition, Nashville, USA, Vol. 6, 1999, p. 33.

[21] M. MacDiarmid, M. Bacic, R. Daniel, Extension and application of a novel hardware-in-the-loop simulator design methodology, in: Decision and Control, 2008. CDC 2008. 47th IEEE Conference on, IEEE, 2008, pp. 5054-5061. 


\section{List of Figures}

$1 \quad$ Structure of AMS $\ldots \ldots \ldots \ldots \ldots \ldots \ldots$

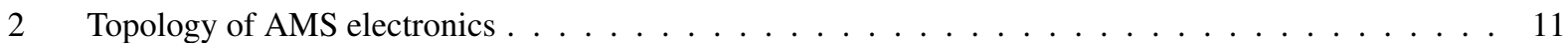

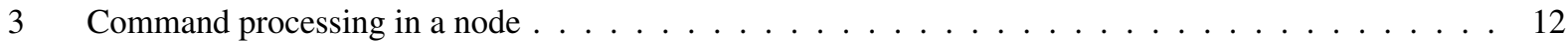

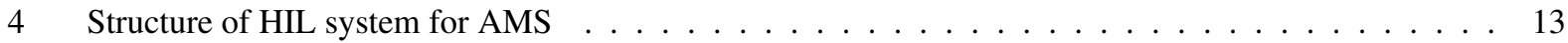

$5 \quad$ Simulation software $\ldots \ldots \ldots \ldots \ldots \ldots \ldots \ldots$

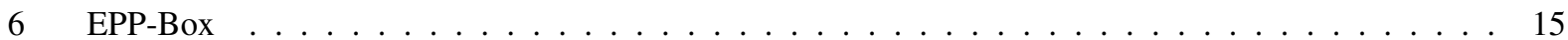

7 General behavior model for AMS nodes . . . . . . . . . . . . . . . . . . . . . . . . 16

$8 \quad$ A sample of the descriptive language . . . . . . . . . . . . . . . . . . . . . . . . . . . . 17

9 Data flow of the HIL system . . . . . . . . . . . . . . . . . . . . . . 18

10 Running the simulation software . . . . . . . . . . . . . . . . . . . . . . . . . . . 19

11 Tracker thermal control system . . . . . . . . . . . . . . . . . . . . . . . . . . . 20

12 Reproduce space environment on the flight simulator . . . . . . . . . . . . . . . . . . . . 21

13 Generate different conditions for single sensors . . . . . . . . . . . . . . . . . . . . . . 22

14 Generate combined conditions . . . . . . . . . . . . . . . . . . . . . 23

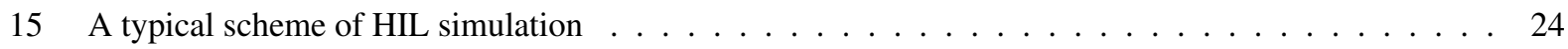

16 Scheme of HIL simulation for AMS . . . . . . . . . . . . . . . . . . . . . . . . . . 25

17 Closed-loop scheme of HIL simulation for complex systems . . . . . . . . . . . . . . . . 26 


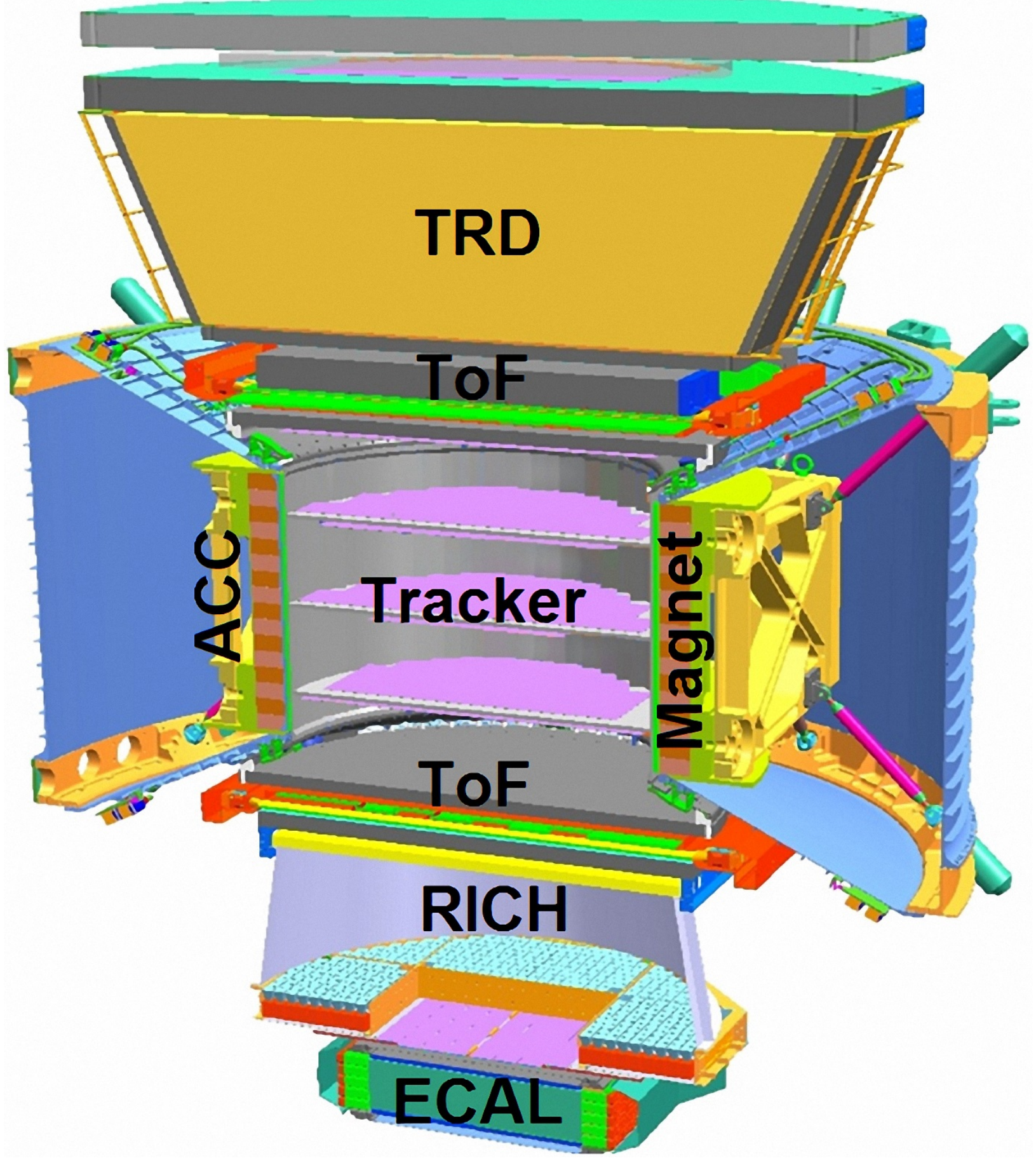

Figure 1: Structure of AMS 


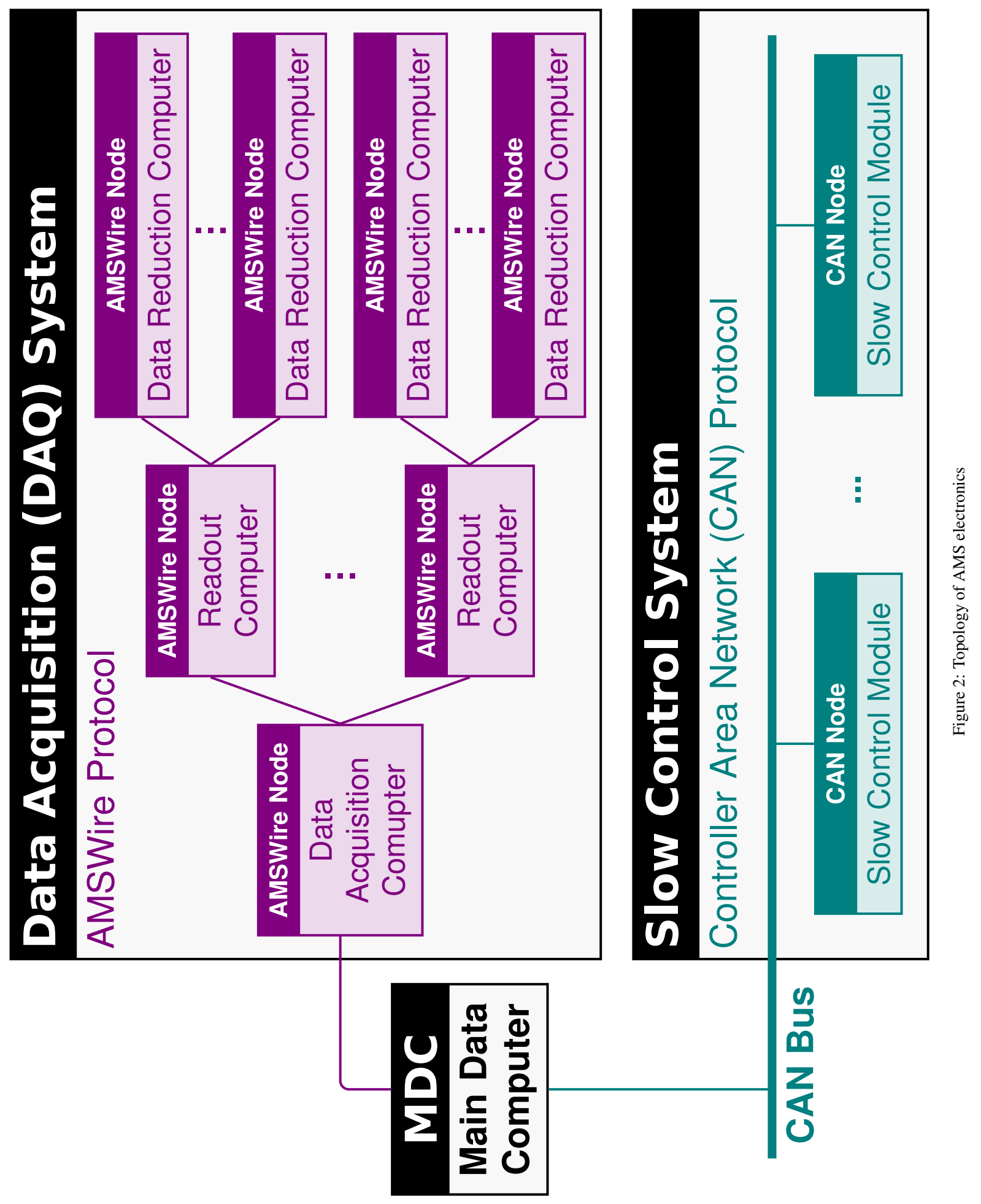




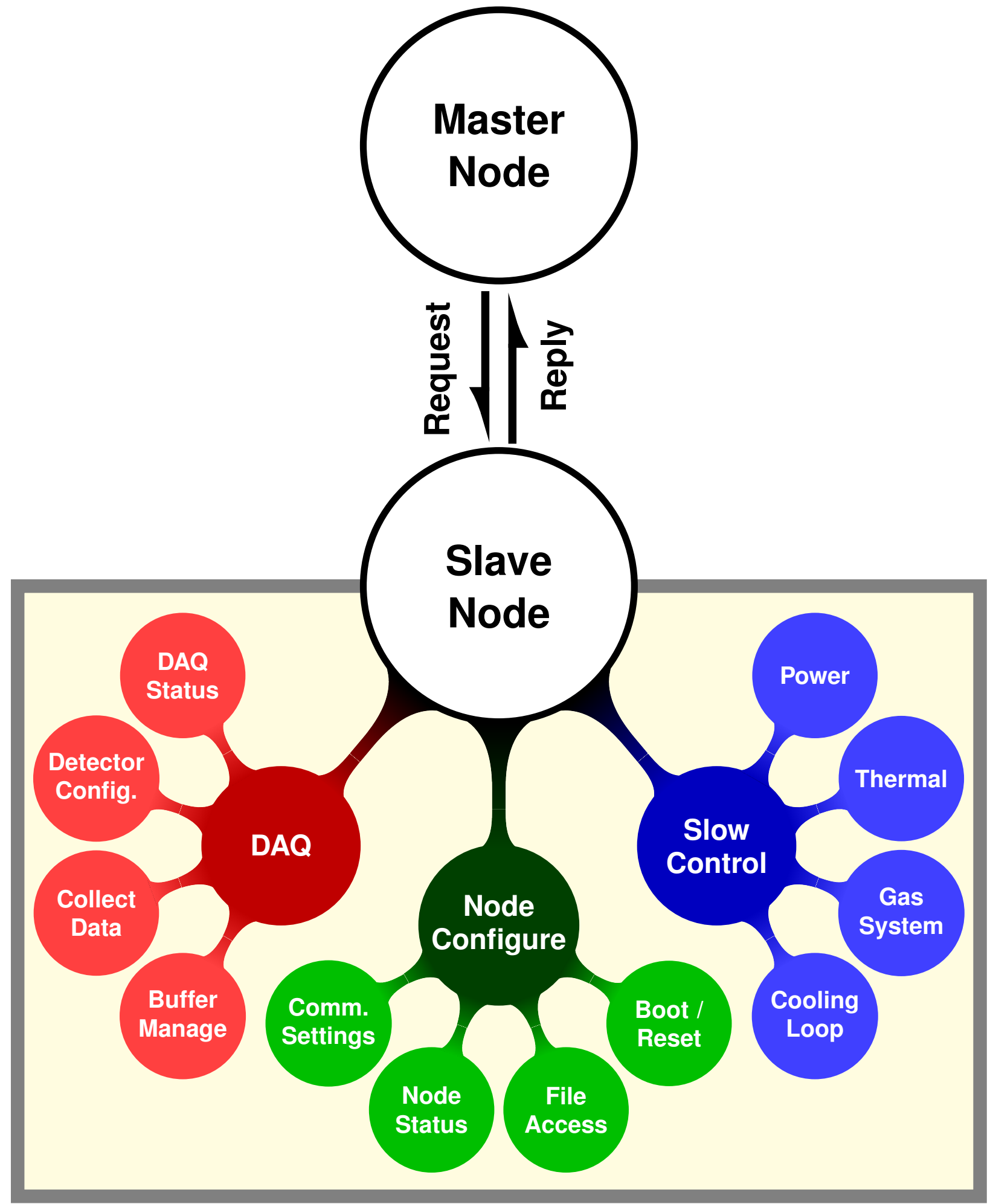

Figure 3: Command processing in a node 


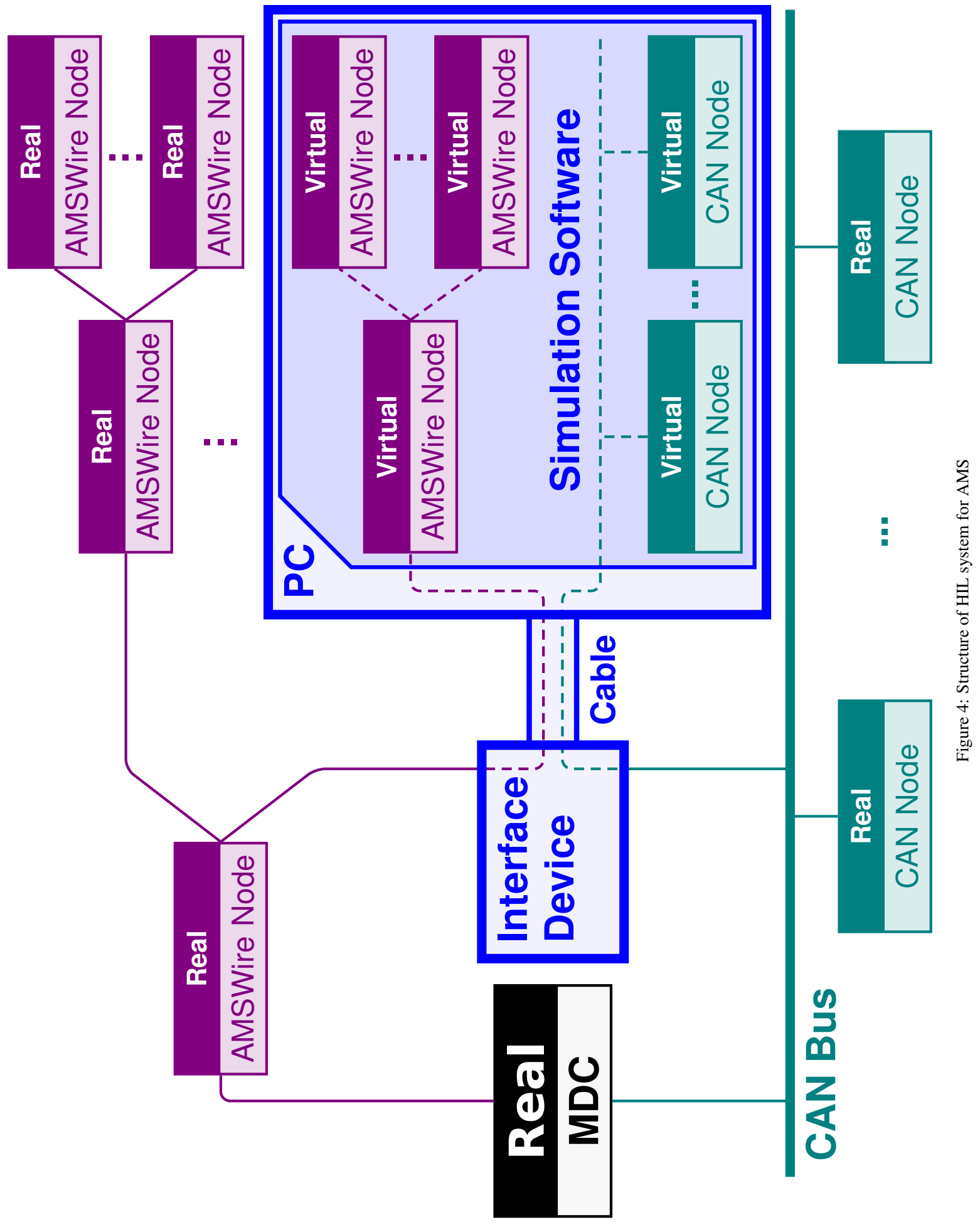




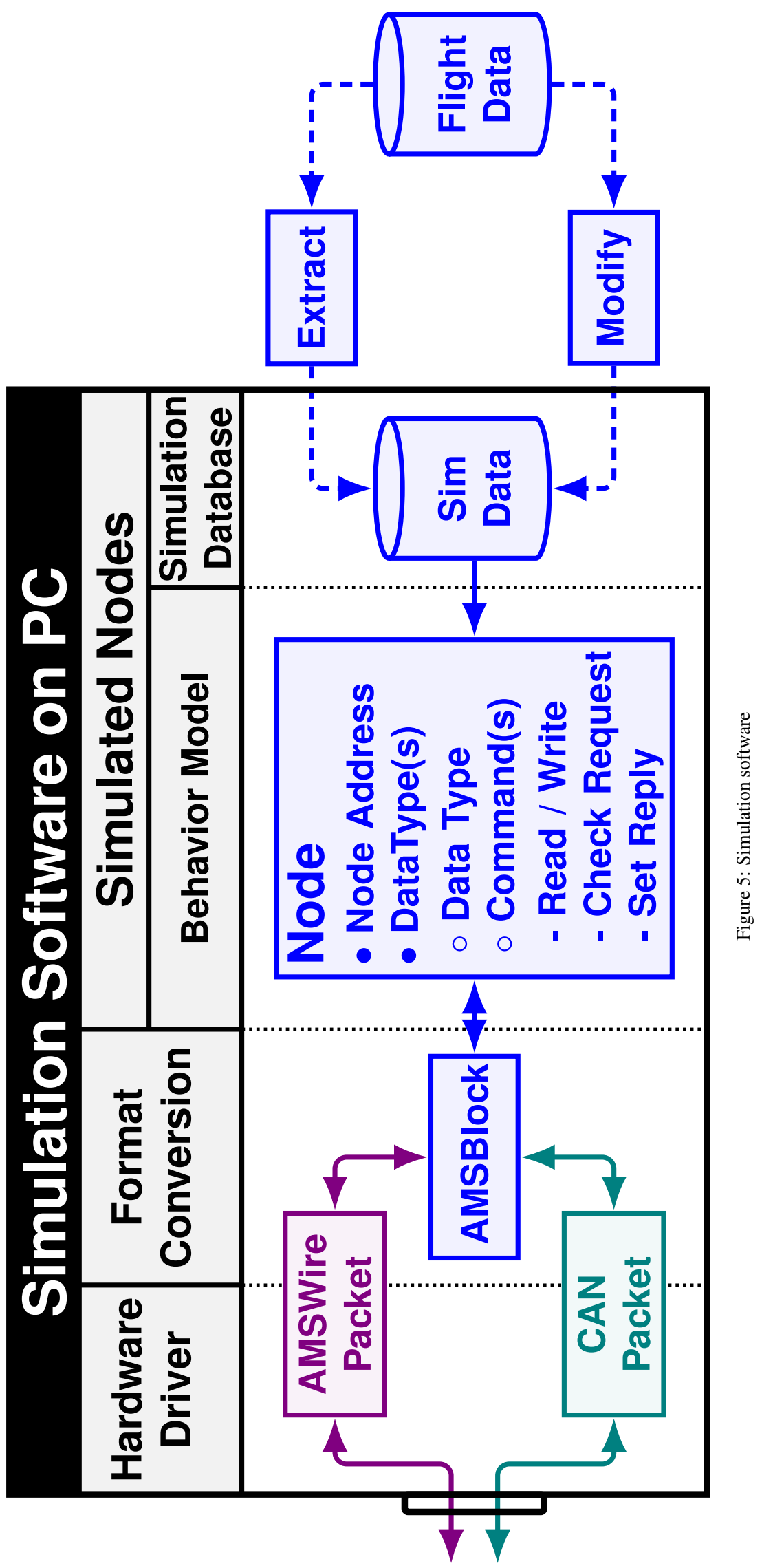


Figure 6

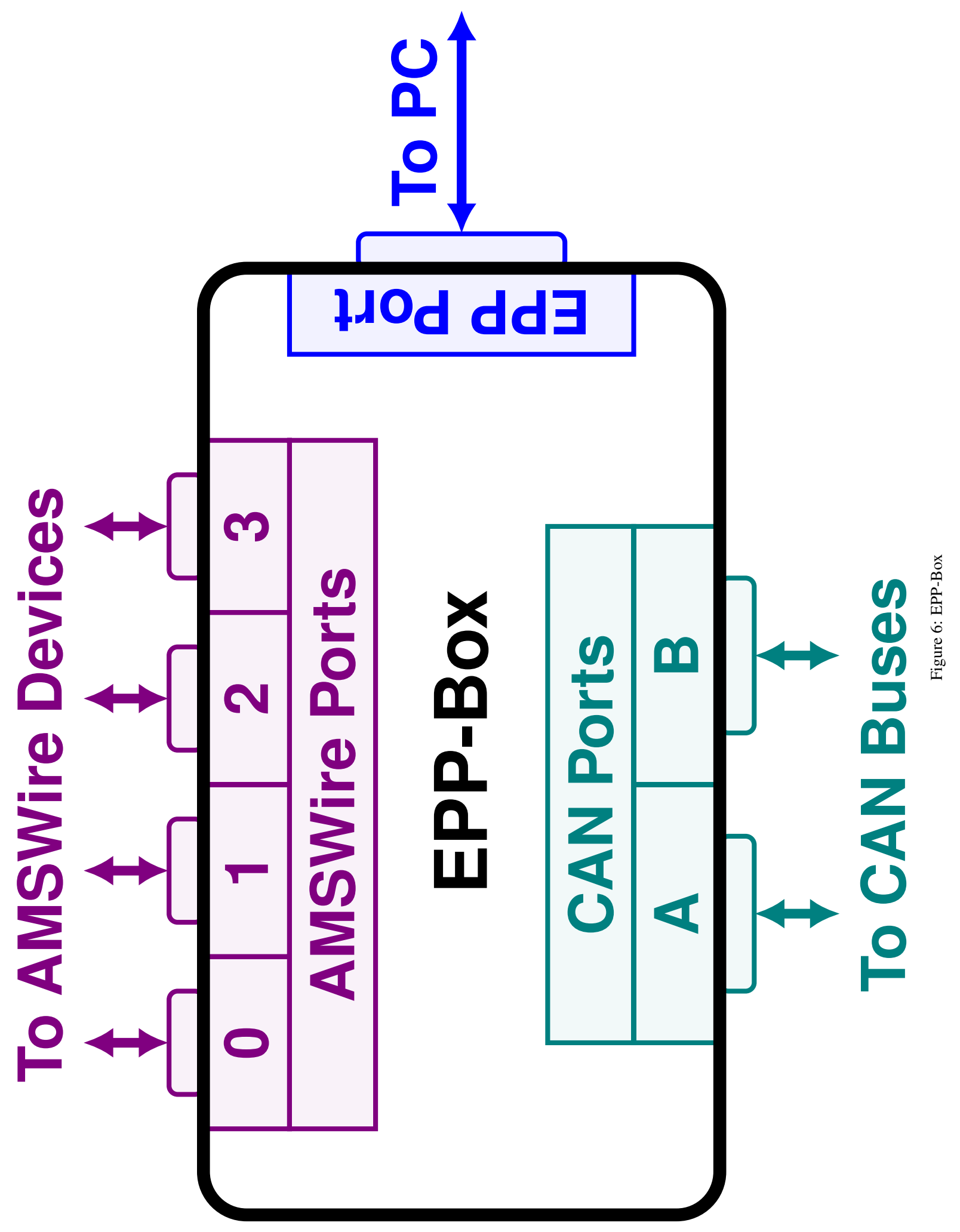




\section{Node}

\section{NodeAddress}

\section{DataType}

\section{DataType Code}

\section{Command}

\section{Read / Write}

Request (Filter Criteria)

\section{Reply (Data Settings)}

\section{- - More Command(s)}

- - More DataType(s)

Figure 7: General behavior model for AMS nodes 


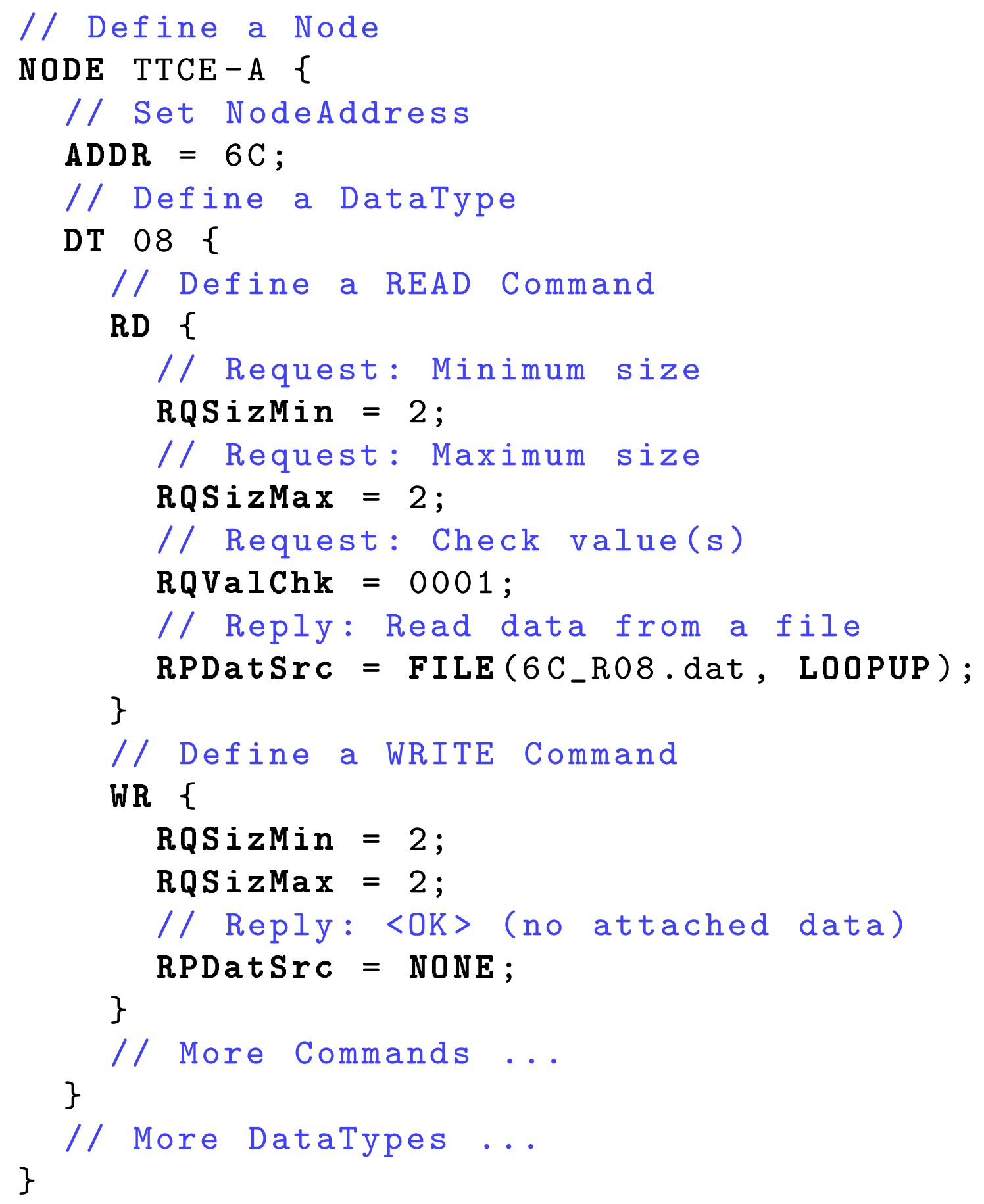


Figure 9

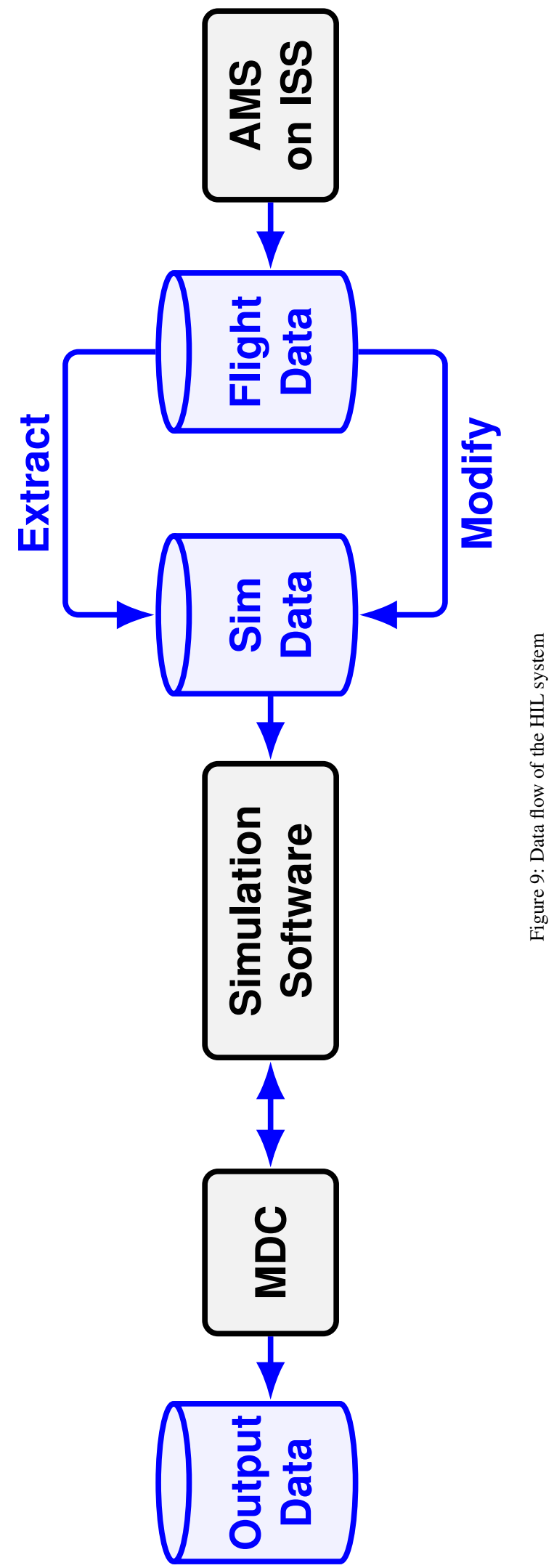


= = = = = = = = = = = = = = = =

\#1 Parse Config File

$==============$

Node: $\quad$ TTCE -A

- 1st Node

+-Address: $06 \mathrm{C}$

- NodeAddress

+- Type :

$\mathrm{CAN}$

- Protocol

$+-\mathrm{Dat}$ aType :

$$
\begin{aligned}
\text { 00000A } 00001800001900001 \mathrm{~A} \\
\\
-- \text { Defined DataType }
\end{aligned}
$$

Node :

TTCE - B

- 2nd Node

+-Address : 06D

+- Type: $\quad$ CAN

+-DataType : 000001000006000007000008000009 $00000 \mathrm{~A} \quad 00001800001900001 \mathrm{~A}$

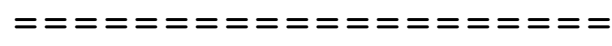

\#2 Start Simulation

$=============$

Initializing EPP device... -- Initialization EPP device initialized.

(Press 'q' to quit)

[RCV]: $01 \quad 12 \quad 34 \quad 56 \quad 78$

- Received Data

[SIM ]: TTCE-A

- Responding Node

[SIM]: PING mode

- Behavior(Ping)

[REQ]: $[\mathrm{R} \quad \mathrm{NA}=06 \mathrm{C} \quad \mathrm{DT}=000001 \mathrm{DC}=4] 12345678$

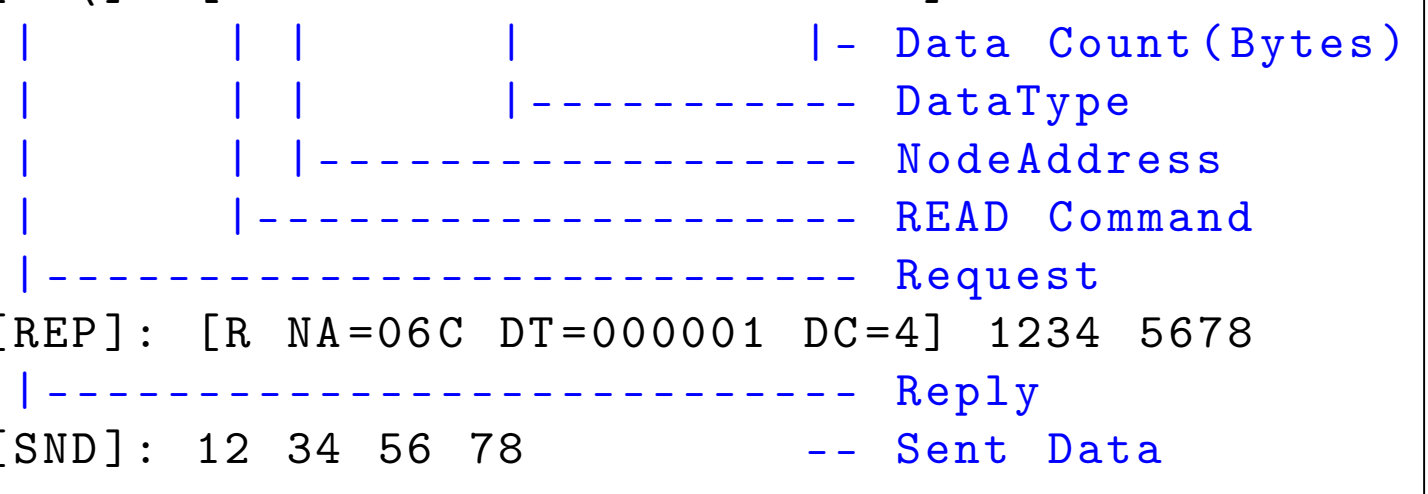

Figure 10: Running the simulation software 


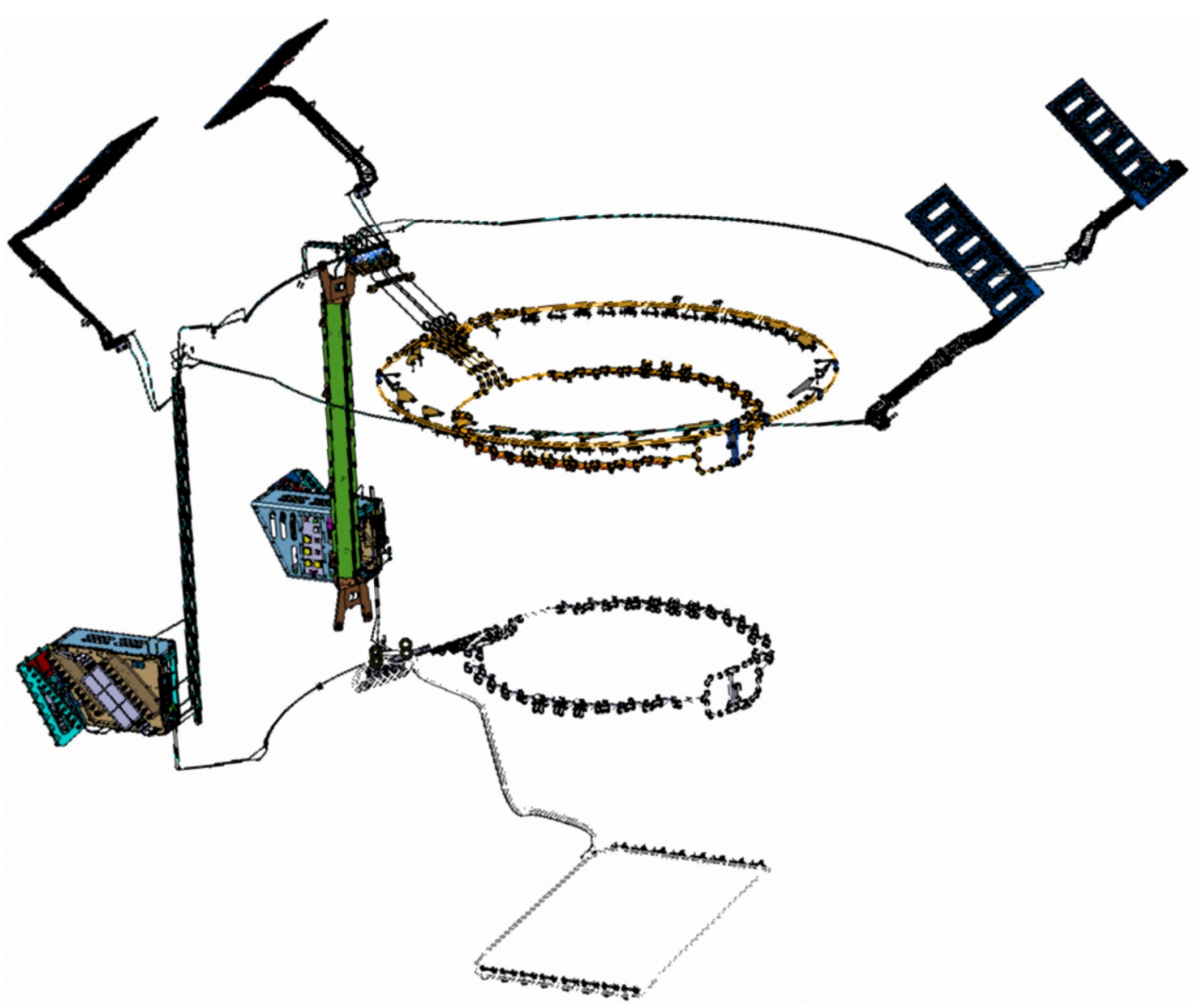

Figure 11: Tracker thermal control system 


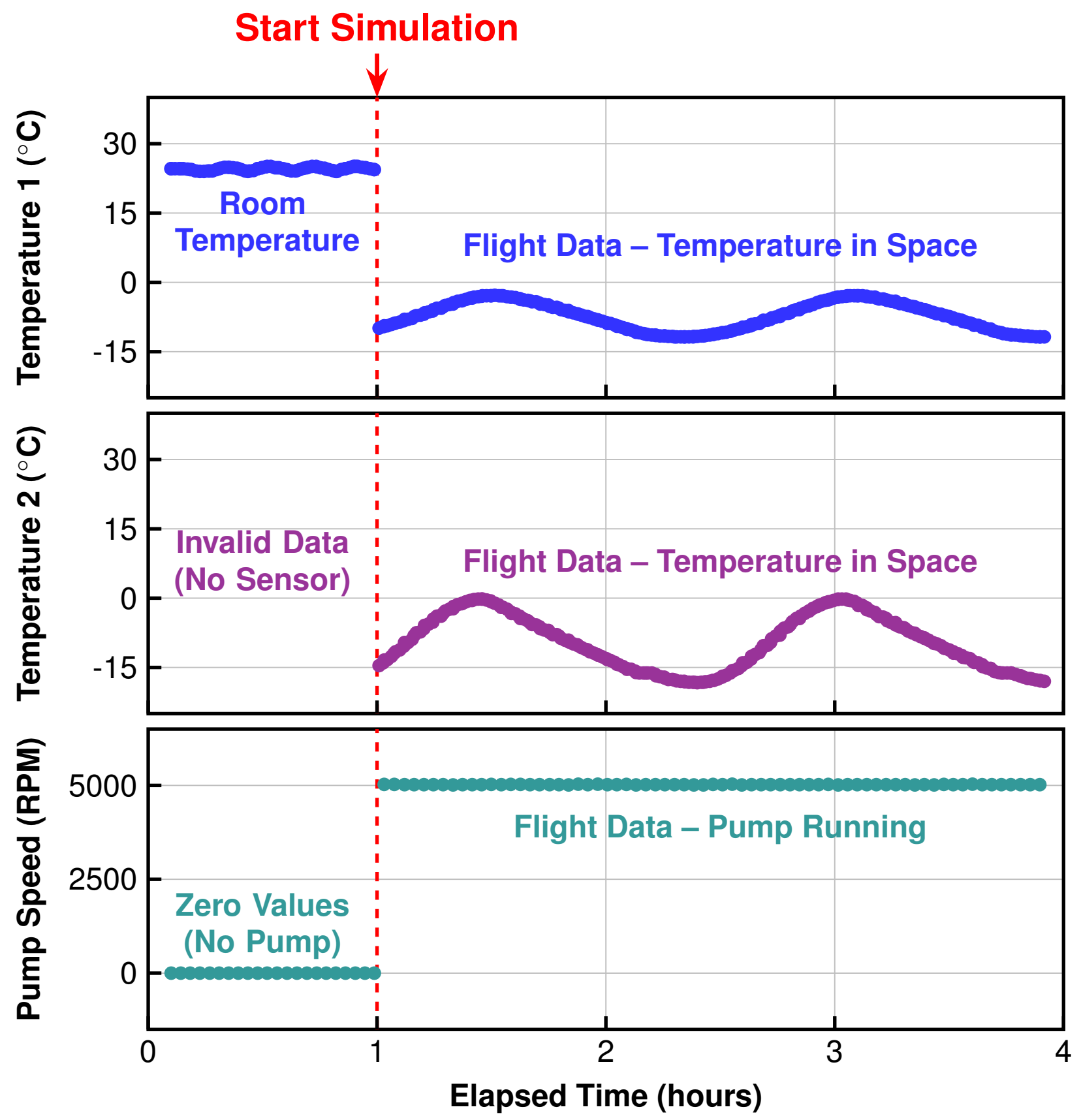

Figure 12: Reproduce space environment on the flight simulator 


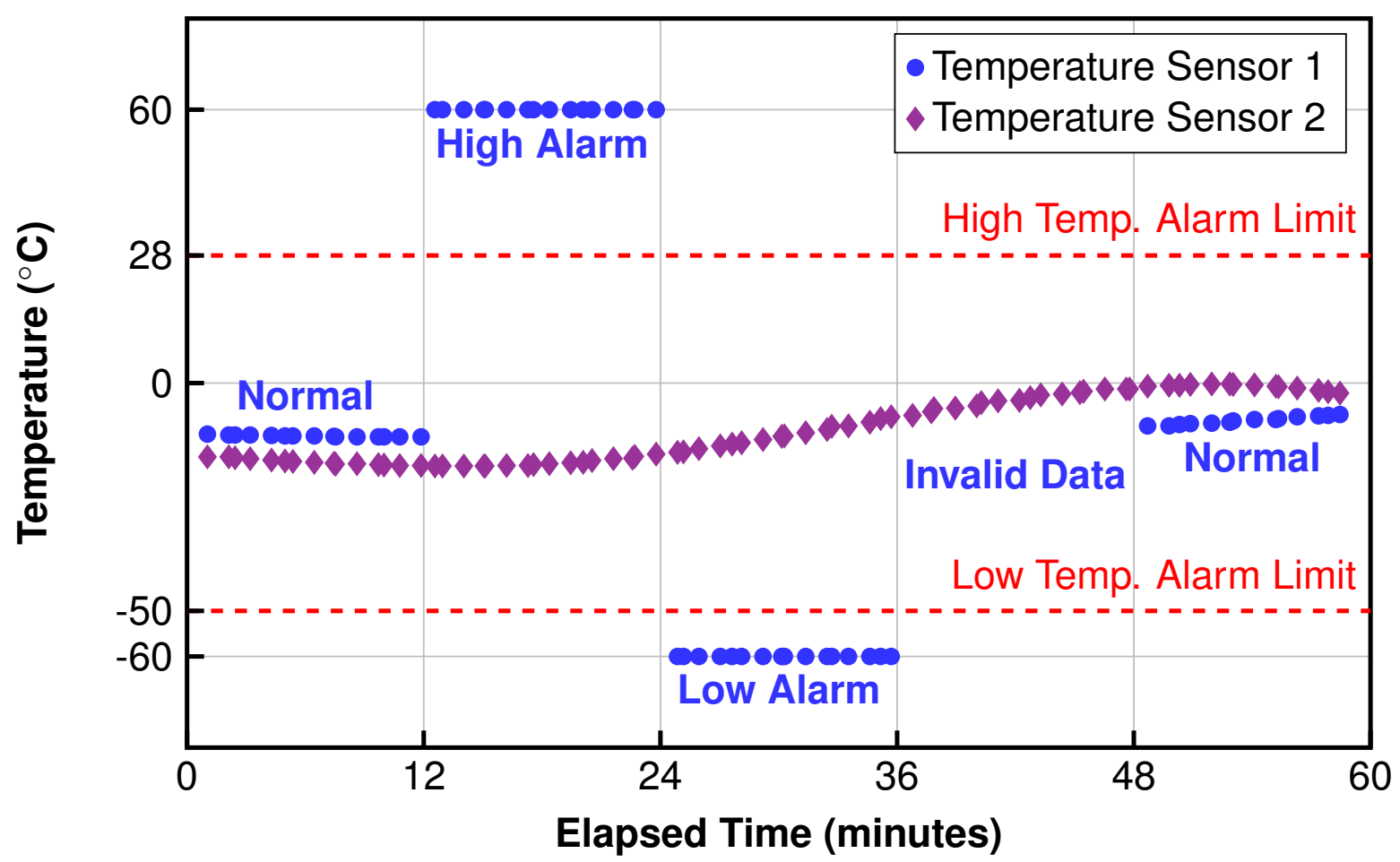

(a) Temperature sensors

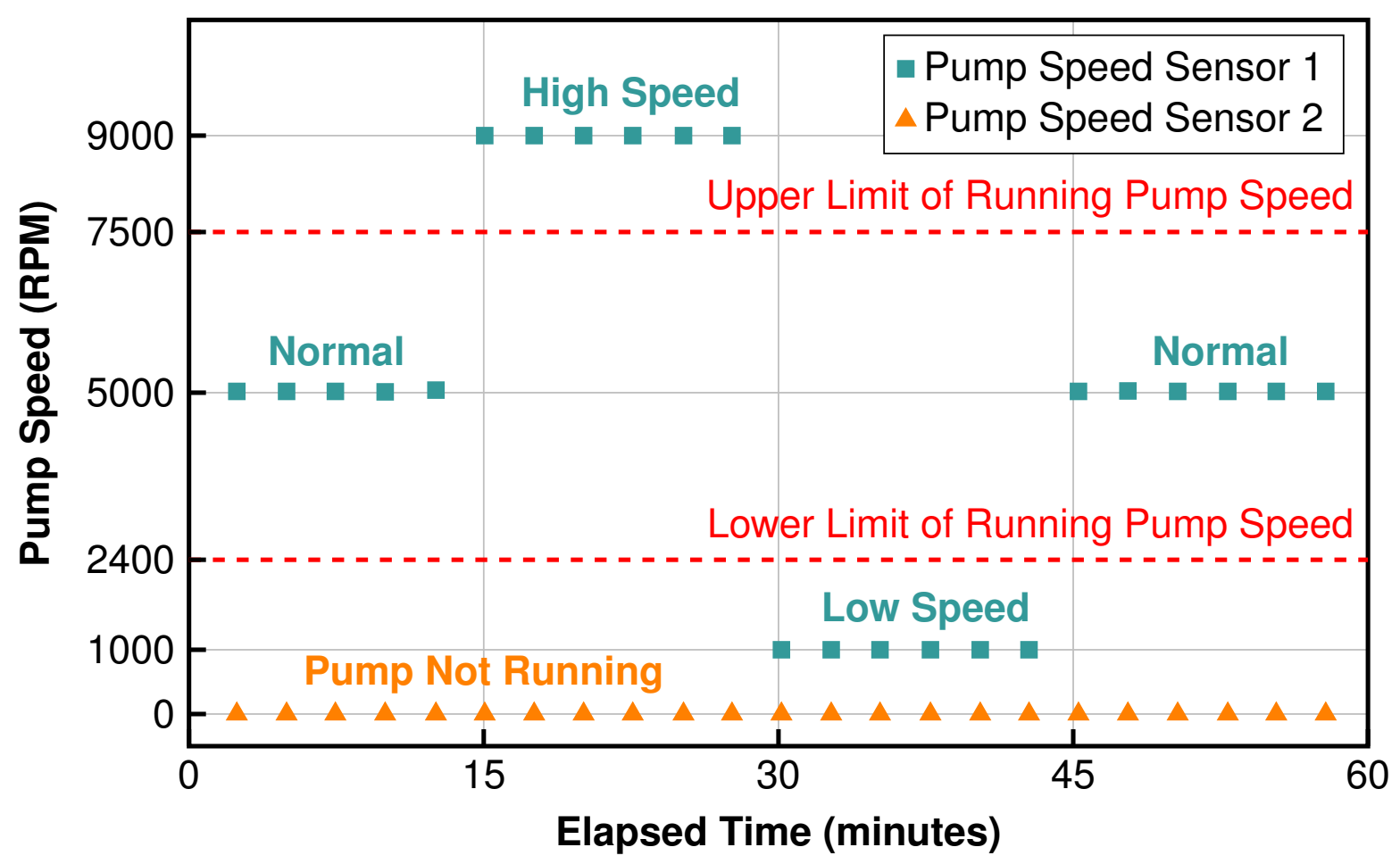

(b) Pump speed sensors

Figure 13: Generate different conditions for single sensors 


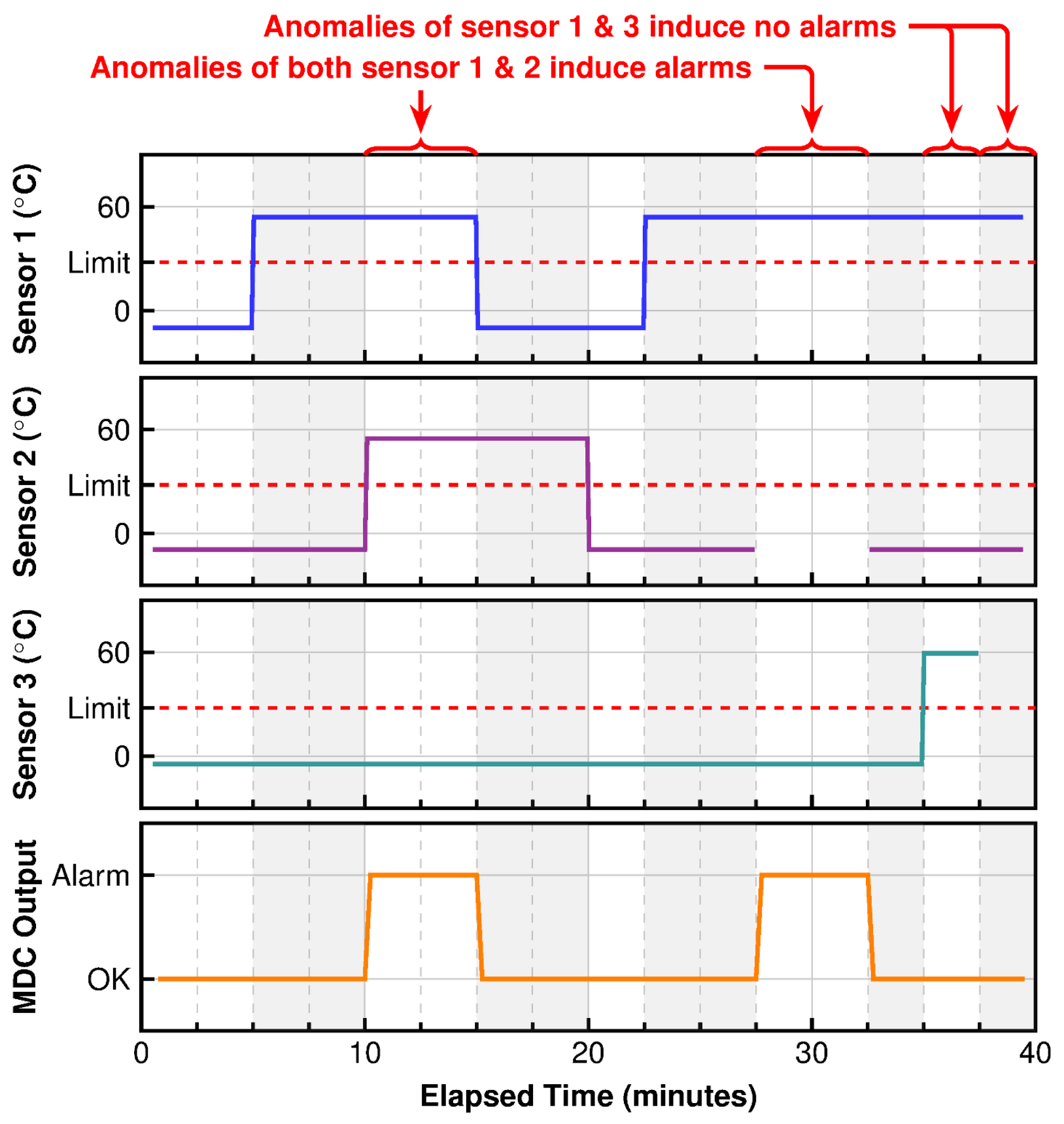

Figure 14: Generate combined conditions 


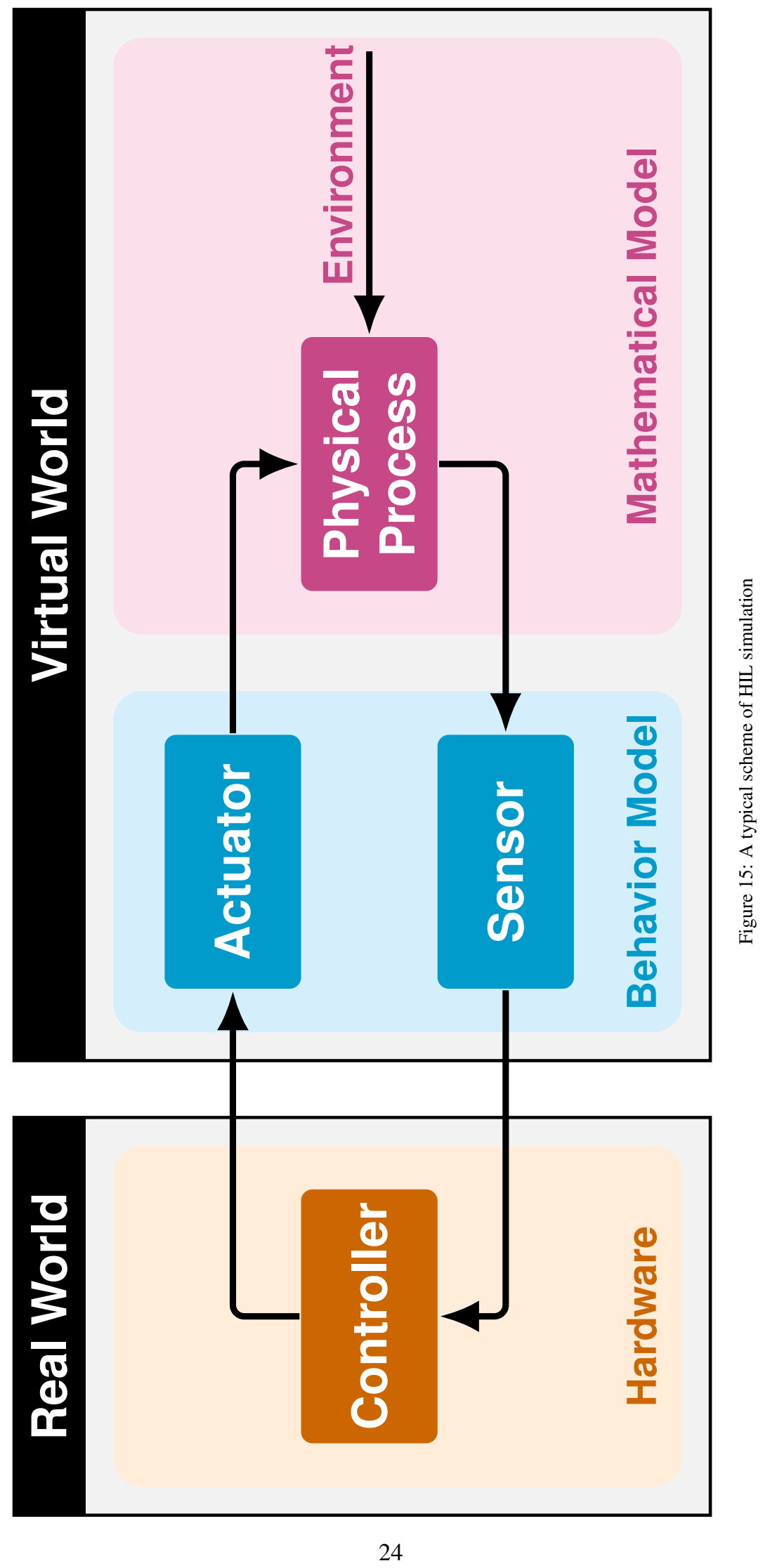




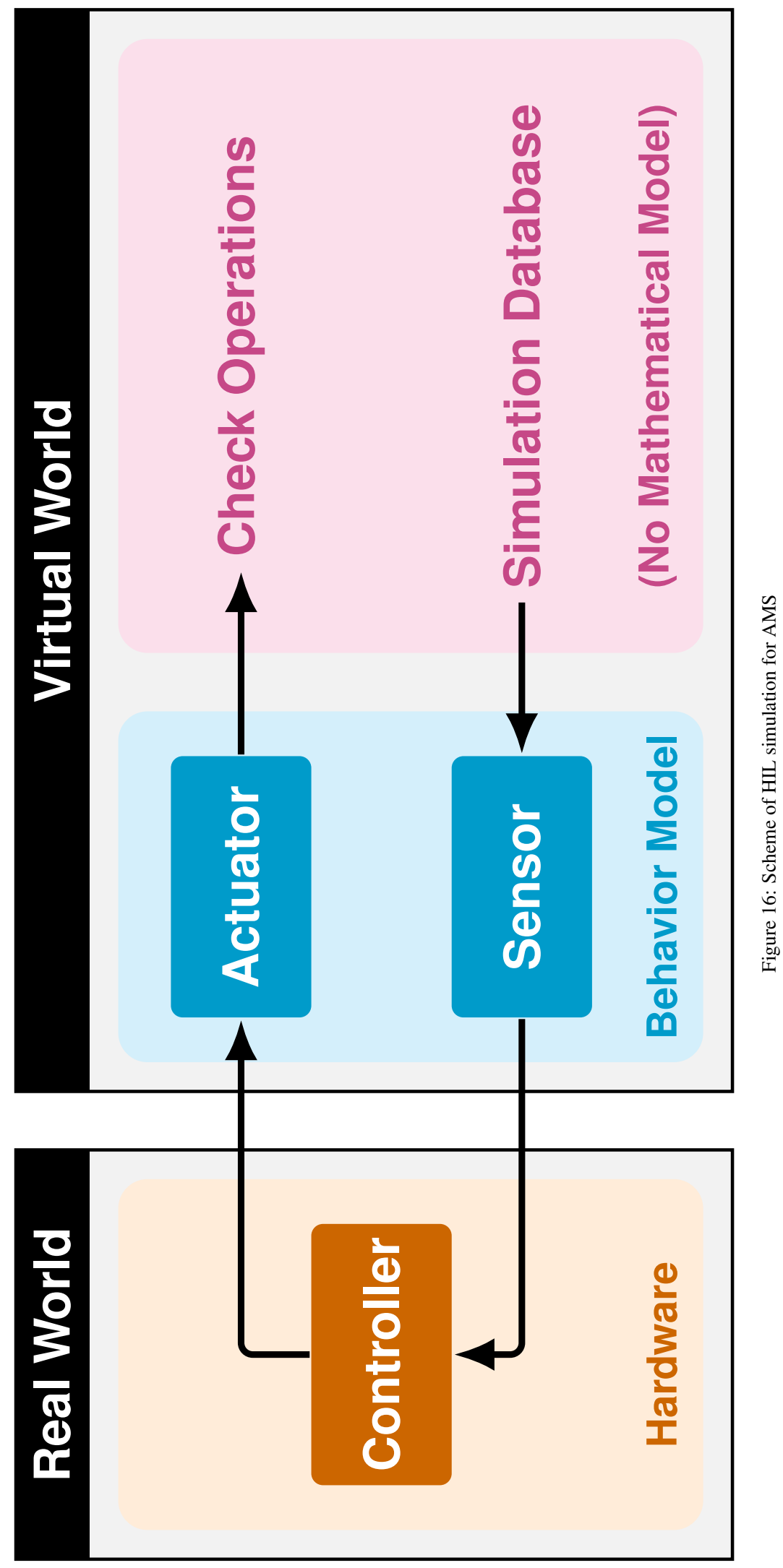




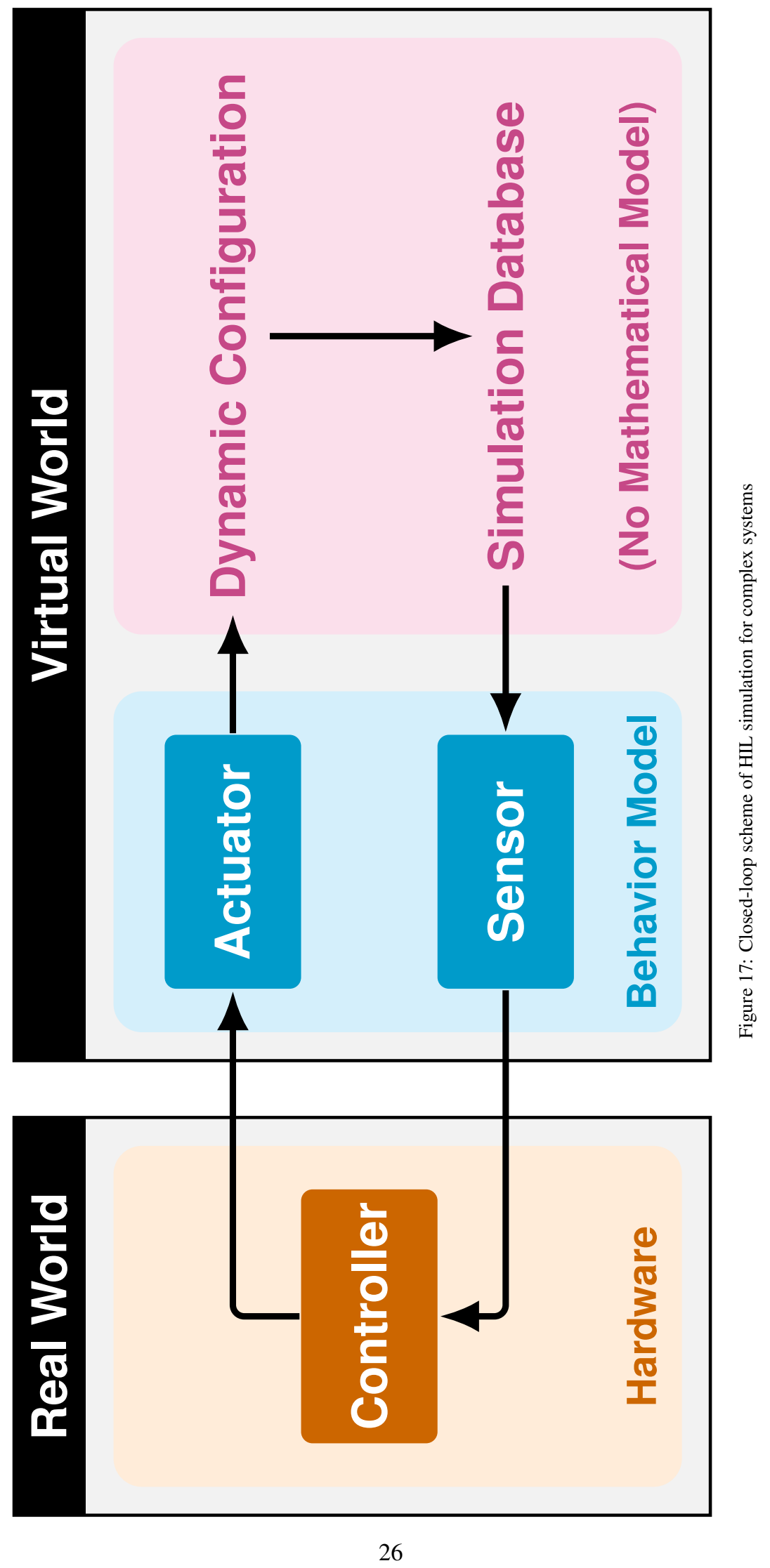

